\title{
RAID 5 Installations on Linux and Creating File System
}

\author{
Puri Ganesh D. \\ Assistant Professor \\ AVCOE Sangamner. \\ A'Nagar Maharashtra
}

\author{
Puri Dinesh D. \\ Assistant Professor \\ SSBTCOE Bambhori \\ Jalgaon Maharashtra
}

\author{
Wackchaure Manoj A. \\ Assistant Professor \\ AVCOE Sangamner. \\ A'Nagar Maharashtra
}

\begin{abstract}
Redundant Array of Independent (originally Inexpensive) Disks or RAID can be set up using hardware or software. Hardware RAID is more expensive, but offers better performance. Software RAID is cheaper and easier to manage, but it uses your CPU and your memory. Where ten years ago nobody was arguing about the best choice being hardware RAID, this has changed since technologies like mdadm, lvm and even zfs focus more on manageability. The workload on the CPU for software RAID used to be high, but CPU's have gotten a lot faster. [1]
\end{abstract}

\section{INTRODUCTION}

RAID is basically for two things. First is to increase the performance by striping data across multiple drives, thus it can allow multiple drives to supply or take data stream simultaneously. Second it can replicate data across multiple drives to supply so it reduces the risk associated with single failed disk. It assumes two things. First one is mirroring in which data blocks are reproduced bit for bit on several different drives, and parity schemes in which one or more drives contain an error-correcting check-sum of the blocks on the remaining data drives.

\section{SOFTWARE RAID 5}

Linux requires you to create one or more partitions. A partition's geometry and size is usually defined by a starting and ending cylinder(sometimes by sector). Partitions can be of type primary (maximum four), extended(maximum one) or logical (contained within the extended partition). Each partition has a type field that contains a code. This determines the computers operating system or the partitions file system.[2]

Table 2.1 Partition's type and naming

\begin{tabular}{|l|l|}
\hline Partition Type & naming \\
\hline Primary $(\max 4)$ & $1-4$ \\
\hline Extended $(\max 1)$ & $1-4$ \\
\hline Logical & 5 \\
\hline
\end{tabular}

The hard disk devices are named /dev/hdx or $/ \mathrm{dev} / \mathrm{sdx}$ with $\mathrm{x}$ depending on the hardware configuration. Next is the partition number, starting the count at 1 . Hence the four (possible) primary partitions are numbered 1 to 4 . Logicalpartition counting always starts at 5 . Thus $/ \mathrm{dev} / \mathrm{hda} 2$ is the second partition on the first ATA hard disk device, and /dev/hdb5 is the first logical partition on the second ATA hard disk device. Same for SCSI, /dev/sdb3 is the third partition on the second SCSI disk.
Table 2.2 Partition's name and device

\begin{tabular}{|l|l|}
\hline Partition & device \\
\hline$/ \mathrm{dev} / \mathrm{hda} 1$ & first primary partition on /dev/hda \\
\hline$/ \mathrm{dev} / \mathrm{hda} 2$ & $\begin{array}{l}\text { second primary or extended partition } \\
\text { on } / \mathrm{dev} / \mathrm{hda}\end{array}$ \\
\hline$/ \mathrm{dev} / \mathrm{sda} 5$ & first logical drive on $/ \mathrm{dev} / \mathrm{sda}$ \\
\hline$/ \mathrm{dev} / \mathrm{sdb} 6$ & second logical drive on $/ \mathrm{dev} / \mathrm{sdb}$ \\
\hline
\end{tabular}

For software RAID we require physical partitions with equal size. For ex. We take sdb disk which is partitioned into $\mathrm{sdb} 1, \mathrm{sdb} 2, \mathrm{sdb} 3$ of equal size. we create a partition with fdisk on /dev/sdb.[3] First we start the fdisk tool with dev/sdb as argument. While creating partitions care should be taken not to partition the wrong disk. Device contains neither a valid DOS partition table, nor Sun, SGI. Building a new DOS disk label. Changes will remain in memory only, until you decide to write them. After that, of course, the previous content won't be recoverable. Inside the fdisk tool, we can issue the $p$ command to see the current disks partition. After restoring a master boot record with dd, we need to force the kernel to reread the partition table with partprobe. After running partprobe, the partitions can be used again.

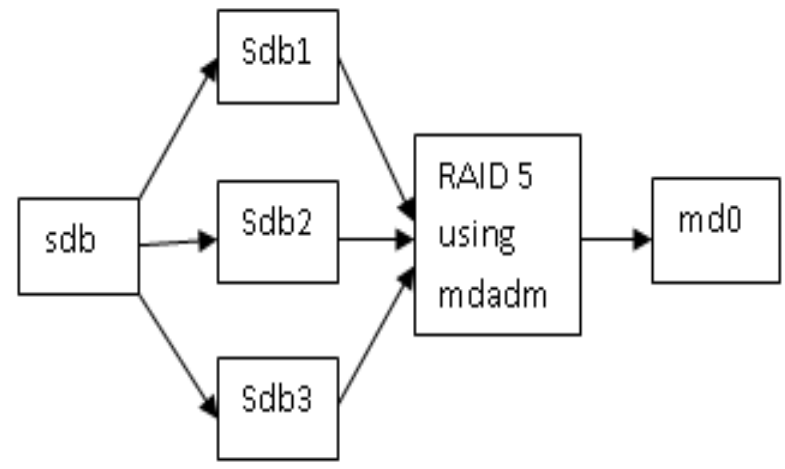

Fig 1: Software RAID-5 using mdadm

In the above fig. 1 software RAID-5 is shown which is basically for mirroring as well as performance. In this three physical drives of single SCSI disk are used. Using fdisk tool in linux sdb is partitioned into physical parts. Using mdadm tool RAID-5 level is created. The array of physical disks is created as md0. In software RAID existing disk and CPU is used. It can be extended for next levels of RAID.[4]

\section{INSTALLATION OF RAID 5}

For Hardware RAID 5 we require to disks of specific configuration for ex. three hard disks of $500 \mathrm{~GB}$ size each. It is expensive to get such disks. For experimental purpose we will take USB disks of small size and extend the experiment 
for large size. In this installation two USB disks of size 2.4GB are taken for experiment.[5]

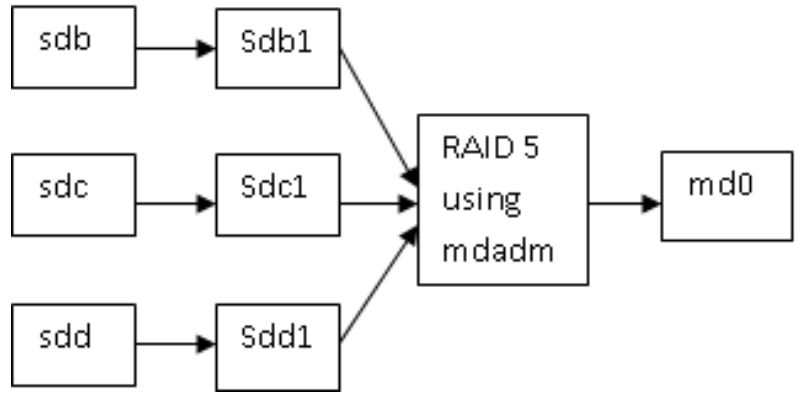

Fig 2: Procedure for RAID-5 using mdadm

In above fig 2 three disks are partitioned. First partition of each disk is of size 2.4 GB. Steps for partitioning are given below. Results are obtained on fedora 18 os and core 2 Duo 64 bit machine.

\subsection{Steps for Installation}

\subsubsection{Using fdisk for partition}

[root@CGLAB ]\# fdisk /dev/sdb

Welcome to fdisk (util-linux 2.22.1).

Changes will remain in memory only, until you decide to write them.[6]

Be careful before using the write command.

Command ( $m$ for help): $n$

Partition type:

p primary (1 primary, 0 extended, 3 free)

e extended

Select (default $\mathrm{p}): \mathrm{p}$

Partition number (1-4, default 2): 1

Partition 1 is already defined. Delete it before re-adding it.

Command ( $m$ for help): $\mathrm{d}$

Selected partition 1

Partition 1 is deleted

Command ( $m$ for help): $\mathrm{n}$

Partition type:

p primary ( 0 primary, 0 extended, 4 free $)$

e extended

Select (default p): $\mathrm{p}$

Partition number (1-4, default 1): 1

First sector (2048-15820799, default 2048):

Using default value 2048

Last sector, +sectors or +size $\{\mathrm{K}, \mathrm{M}, \mathrm{G}\}$ (2048-15820799, default 15820799): 5000000

Partition 1 of type Linux and of size $2.4 \mathrm{GiB}$ is set.

In the same way partition for second disk sdc can be created of size $2.4 \mathrm{~GB}$.

\subsubsection{Change Partition type fd}

The next step is to create a partition of type fd on every disk. The fd type is to set the partition as Linux RAID auto detect.

Command ( $\mathrm{m}$ for help): $\mathrm{t}$

Selected partition 1

Hex code (type L to list codes): fd

Changed system type of partition 1 to fd (Linux raid autodetect)

Command ( $\mathrm{m}$ for help): w

The partition table has been altered!

Calling ioctl() to re-read partition table.

WARNING: Re-reading the partition table failed with error 16: Device or resource busy.

The kernel still uses the old table. The new table will be used at the next reboot or after you run partprobe(8) or kpartx(8)
Syncing disks.

\subsection{3 verify all partitions}

Now all disks are ready for raid 5, so we have to tell the system what to do with these disks.

[root@CGLAB ]\# fdisk -1 2>/dev/null | grep raid

/dev/sdb1 $2048 \quad 5000000 \quad 2498976+$ fd Linux raid

autodetect

$/$ dev/sdc1 $2048 \quad 5000000 \quad 2498976+$ fd Linux raid autodetect

\subsubsection{Create the RAID 5}

Issue the command mdadm with the correct parameters. [root@CGLAB ]\# mdadm --create /dev/md0 --chunk=64 -level $=5$--raid-devices $=2 / \mathrm{dev} / \mathrm{sdb} 1 / \mathrm{dev} / \mathrm{sdc} 1$

mdadm: /dev/sdc1 appears to be part of a raid array:

level $=$ raid 0 devices $=0$ ctime $=$ Thu Jan 1 05:30:00 1970

mdadm: partition table exists on /dev/sdc1 but will be lost or meaningless after creating array

Continue creating array? y

mdadm: Defaulting to version 1.2 metadata

mdadm: array /dev/md0 started.

\subsubsection{How fdisk -l sees RAID 5}

[root@CGLAB ]\# fdisk -1/dev/md0

Disk /dev/md0: 2556 MB, 2556821504 bytes, 4993792 sectors

Units $=$ sectors of $1 * 512=512$ bytes

Sector size (logical/physical): 512 bytes / 512 bytes

I/O size (minimum/optimal): 65536 bytes / 65536 bytes.

\subsubsection{Check Entry in mdstat}

[root@CGLAB ]\# cat/proc/mdstat

Personalities : [raid6] [raid5] [raid4]

md0 : active raid5 sdc1[2] sdb1[0]

2496896 blocks super 1.2 level 5, 64k chunk, algorithm 2 [2/1] [U_]

$[==============>$...... $] \quad$ recovery $=74.1 \%$ $(1851300 / 2496896)$ finish $=2.1 \mathrm{~min}$ speed $=5056 \mathrm{~K} / \mathrm{sec}$ unused devices: <none>

\subsubsection{Detail information on active RAID 5 Device} [root@CGLAB ]\# mdadm --detail /dev/md0

$/ \mathrm{dev} / \mathrm{md} 0$ :

Version : 1.2

Creation Time: Wed Nov 27 15:19:40 2013

Raid Level : raid5

Array Size : 2496896 (2.38 GiB $2.56 \mathrm{~GB})$

Used Dev Size : 2496896 (2.38 GiB 2.56 GB)

Raid Devices : 2

Total Devices : 2

Persistence : Superblock is persistent

Update Time : Wed Nov 27 15:28:01 2013

State : clean

Active Devices : 2

Working Devices : 2

Failed Devices : 0

Spare Devices : 0

Layout : left-symmetric

Chunk Size : 64K

Name : CGLAB:0 (local to host CGLAB)

UUID : 572e8de9:f27814e0:8800be41:1061c424

Events : 18

Number Major Minor RaidDevice State

$\begin{array}{llllll}0 & 8 & 17 & 0 & \text { active sync } & / \mathrm{dev} / \mathrm{sdb} 1\end{array}$

$\begin{array}{lllll}2 & 8 & 33 & 1 & \text { active sync } / \mathrm{dev} / \mathrm{sdc} 1\end{array}$ 


\section{CREATING FILE SYSTEM ON RAID5}

\subsection{Common file systems}

\subsection{1 ext 2 and ext 3}

Once the most common Linux file systems is the ext2 (the second extended) file system. A disadvantage is that file system checks on ext2 can take a long time. So ext2 is being replaced by ext3 on most Linux machines. They are essentially the same, except for the journaling which is only present in ext3. Journaling means that changes are first written to a journal on the disk. The journal is flushed regularly, writing the changes in the file system. Journaling keeps the file system in a consistent state, so we don't need a file system check after an unclean shutdown or power failure.

we can create these file systems with the $/ \mathrm{sbin} / \mathrm{mkfs}$ or $/ \mathrm{sbin} / \mathrm{mke} 2 \mathrm{fs}$ commands. Use mke $2 \mathrm{fs}-\mathrm{j}$ to create an ext 3 file system. We can convert an ext 2 to ext 3 with tune $2 \mathrm{fs}-\mathrm{j}$. We can mount an ext 3 file system as ext 2 , but then we lose the journaling. So it need to run mkinitrd if we are booting from this device.[1][2]

\subsection{2 ext4}

Since 2008 the newest incarnation of the ext file system is ext4 is available in the Linux kernel. ext4 support larger files (up to 16 terabyte) and larger file systems than ext3 (and many more features).[7]

\subsubsection{Vfat}

The vfat file system exists in a couple of forms : fat 12 for floppy disks, fat 16 on ms-dos, and fat32 for larger disks. The Linux vfat implementation supports all of these, but vfat lacks a lot of features like security and links. The fat disks can be read by every operating system, and are used a lot for digital cameras, USB sticks and to exchange data between different OS on a home user's computer.[7]

\subsection{Creating ext4 file system on RAID 5}

Now we put ext4 file system on $/ \mathrm{dev} / \mathrm{md} 0$ which is created for RAID 5

[root@CGLAB ]\# mkfs.ext4 /dev/md0

mke2fs 1.42 .5 (29-Jul-2012)

Filesystem label $=$

OS type: Linux

Block size $=4096(\log =2)$

Fragment size $=4096(\log =2)$

Stride $=16$ blocks, Stripe width $=16$ blocks

156160 inodes, 624224 blocks

31211 blocks $(5.00 \%)$ reserved for the super user

First data block $=0$

Maximum filesystem blocks $=641728512$

20 block groups

32768 blocks per group, 32768 fragments per group

7808 inodes per group

Superblock backups stored on blocks:

32768, 98304, 163840, 229376, 294912

Allocating group tables: done

Writing inode tables: done

Creating journal (16384 blocks): done

Writing superblocks and filesystem accounting information: done

\subsection{Mounting file system on mount point}

We will mount the filesystem on mount point /root/test where contents of RAID drive are visible. Following fig. 3 explain the steps.[5]

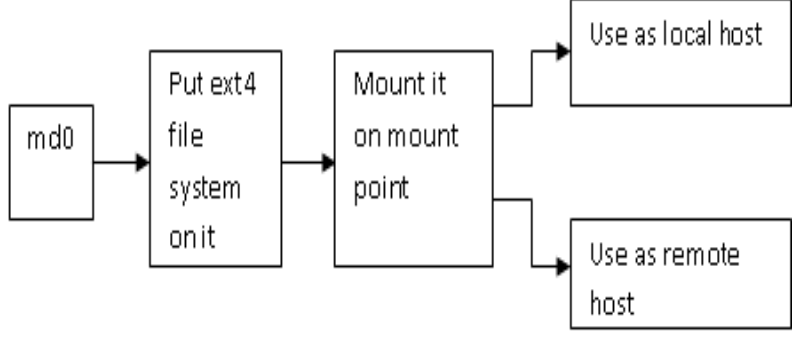

Fig 3: Creating file system and mounting RAID 5

[root@CGLAB ]\# mount /dev/md0/root/test

[root@CGLAB ]\# df -h

Filesystem Size Used Avail Use\% Mounted on

rootfs $50 \mathrm{G} \quad 12 \mathrm{G} \quad 36 \mathrm{G} \quad 25 \% /$

devtmpfs $\quad 927 \mathrm{M} \quad 0927 \mathrm{M} 0 \% / \mathrm{dev}$

tmpfs $938 \mathrm{M} 184 \mathrm{~K} 938 \mathrm{M} 1 \% / \mathrm{dev} / \mathrm{shm}$

tmpfs $\quad 938 \mathrm{M} 2.7 \mathrm{M} 935 \mathrm{M} 1 \% /$ run

tmpfs $\quad 938 \mathrm{M} \quad 0938 \mathrm{M} 0 \% /$ sys/fs/cgroup

/dev/mapper/fedora-root $50 \mathrm{G} \quad 12 \mathrm{G} \quad 36 \mathrm{G} \quad 25 \%$ /

tmpfs $\quad 938 \mathrm{M} 792 \mathrm{~K} 937 \mathrm{M} 1 \% / \mathrm{tmp}$

/dev/sda2 $\quad 485 \mathrm{M} \quad 51 \mathrm{M} \mathrm{410M} 11 \% /$ boot

/dev/mapper/fedora-home 46G 575M 43G 2\%/home

$/$ dev/sdal $\quad 49 \mathrm{G} \quad 16 \mathrm{G} \quad 33 \mathrm{G} \quad 33 \%$

/run/media/root/D6DC85C0DC859AFF

$\begin{array}{llll}/ \mathrm{dev} / \mathrm{md} 0 & 2.4 \mathrm{G} \quad 68 \mathrm{M} \quad 2.2 \mathrm{G} \quad 3 \% / \mathrm{root} / \mathrm{test}\end{array}$

Underlined line shows it is mounted on /root/test

Following step shows how the file system used for storage. So put some files on md0 drive it is visible on mount pt.[6]

[root@CGLAB test1]\# ls

lost+found osa total assignment QP.odt raje.c raj.l

\section{FAULT TOLERANCE IN RAID 5}

We will see what happens when one of the disk failed in RAID 5. In our experiment we will set sdc1 disk faulty and observe the $\log$ status. It continues to work fine but in degraded mode. Following step shows this.

[root@CGLAB ]\# mdadm /dev/md0 -f/dev/sdc1

mdadm: set $/ \mathrm{dev} / \mathrm{sdc} 1$ faulty in $/ \mathrm{dev} / \mathrm{md} 0$

[root@CGLAB ]\# tail /var/log/messages

Nov 27 17:32:04 CGLAB kernel: [13362.597647] md: md0 still in use.

Nov 27 17:32:04 CGLAB kernel: [13362.797729] md: md0 still in use.

Nov 27 17:42:21 CGLAB kernel: [13979.614641] $\mathrm{md} / \mathrm{raid}$ :md0: Disk failure on sdc1, disabling device.

Nov 27 17:42:21 CGLAB kernel: [13979.614641] $\mathrm{md} /$ raid:md0: Operation continuing on 1 devices.

It can be checked in /proc/mdstat file as follows.

[root@CGLAB ]\# cat/proc/mdstat

Personalities : [raid6] [raid5] [raid4]

md0 : active raid5 $\mathrm{sdc} 1[2](\mathrm{F}) \mathrm{sdb} 1[0]$

2496896 blocks super 1.2 level 5, 64k chunk, algorithm 2 [2/1] [U_]

unused devices: <none> 


\subsection{Hot removing faulty disk}

In RAID 5 faulty disks can be hot removed without affecting the operation keeping all data intact on remaining disks in RAID. Following step shows it.[1] [root@CGLAB ]\# mdadm /dev/md0 -r /dev/sdc1 mdadm: hot removed /dev/sdc1 from $/ \mathrm{dev} / \mathrm{md} 0$ [root@CGLAB ]\# cat /proc/mdstat

Personalities : [raid6] [raid5] [raid4] md0 : active raid5 sdb1[0] 2496896 blocks super 1.2 level 5, 64k chunk, algorithm 2 [2/1] [U_]

unused devices: <none>

\subsection{Hot adding disk in RAID 5}

After removing fault we can add disk without affecting operation. We can add other disk of same configuration and same size partition. Following step shows it.[1]

[root@CGLAB ]\# mdadm /dev/md0 -a /dev/sdc1

mdadm: added /dev/sdc1

[root@CGLAB ]\# cat /proc/mdstat

Personalities : [raid6] [raid5] [raid4]

md0 : active raid5 sdc1[2] sdb1[0]

2496896 blocks super 1.2 level 5, 64k chunk, algorithm 2 [2/1] [U_]

$[>\ldots \ldots \ldots \ldots \ldots . . . . . . . . .1 .0 \% \quad$ recovery $=125724 / 2496896)$

finish $=8.0 \mathrm{~min}$ speed $=5144 \mathrm{~K} / \mathrm{sec}$

unused devices: <none>

\section{DRAWBACKS OF RAID 5}

RAID 5 does not replace regular off-line backups. It protects the system against the failure of one disk. It does not protect against the accidental deletion of files. It does not protect against other failures, fires, hackers, or any number of other hazards. Second write performance is not good. Whenever a random block is written, at least one data block and the parity block for that stripe must be updated. The RAID system doesn't know what the new parity block should contain until it has read the old parity block and the old data. Each random write therefore expands into four operations: two reads and two writes.[1]

\section{REFERENCES}

[1] Evi Nemeth., Garth Snyder, Trent R. Hein., Ben Whaley 2012. Unix and Linux System Administration.

[2] Paul Cobbaut. 2013 Linux System Administration.

[3] Maurice J Bach. The design of the UNIX OS

[4] Seymour lipschutz, Marc Lars Lipson 2010 Discrete Mathematics.

[5] Ellis Horowitz, Sartaz Sahni, Sanguthevar Rajasekaran 2007 Fundamentals of computer Algorirhms

[6] Kenneth Rosen, Douglas Host, Rachel Klee, James Farber, Richard Rosinski 2010 The Complete Reference Unix.

[7] Terry Collings, Kurt Wall 2012 Redhat Linux Networking and System Administration. 